\title{
Lizard Skin Patterns and the Ising Model
}

\author{
Szabolcs Zakany $\oplus^{1,2}$ Stanislav Smirnov, ${ }^{3,4,5}$ and Michel C. Milinkovitch $\oplus^{1,2, *}$ \\ ${ }^{1}$ Department of Genetics and Evolution, University of Geneva, 30 quai Ernest-Ansermet, 1211 Genève, Switzerland \\ ${ }^{2}$ SIB Swiss Institute of Bioinformatics, 1211 Geneva, Switzerland \\ ${ }^{3}$ Section of Mathematics, University of Geneva, 7-9 rue du Conseil-Général, 1205 Genève, Switzerland \\ ${ }^{4}$ Skolkovo Institute of Science and Technology, 121205 Moscow, Russia \\ ${ }^{5}$ St. Petersburg University, 199034 St Petersburg, Russia
}

(Received 17 May 2021; accepted 16 December 2021; published 27 January 2022)

\begin{abstract}
The ocellated lizard (Timon lepidus) exhibits an intricate skin color pattern made of monochromatic black and green skin scales, whose dynamics of color flipping are known to be well modeled by a stochastic cellular automaton. We show that the late-time probability distribution of the pattern corresponds to the canonical probability distribution of the antiferromagnetic Ising model and can be generated by dynamics different from the commonly-used Glauber. We comment on skin scale patterns generated by the Ising model on the triangular lattice in the low-temperature limit.
\end{abstract}

DOI: 10.1103/PhysRevLett.128.048102

Introduction.-Skin color patterns are highly similar among individuals within a species; i.e., zebras have stripes, but cheetahs have spots. However, the positional details of the pattern change from one individual to the other. Whereas, in many species, individuals exhibit the same pattern all their life, in others they drastically shift between distinct juvenile and adult patterns. A third category involves the progressive transformation of the juvenile pattern into the adult pattern. The ocellated lizard (Timon lepidus) is such a case: The adult dorsal labyrinthine pattern, made of black and green chains of scales [Fig. 1(a)], is generated through a gradual process of greento-black and black-to-green color switching of individual scales. The switching of a single scale typically takes a few weeks and can happen even 3-4 years after the beginning of the process. The resulting pattern is likely to have a classical camouflaging function based on body outline disruption [2-4].

While skin colour patterning is classically described by Turing's reaction-diffusion (RD) equations [5], at the scale of the ocellated lizard's skin scales these dynamics are effectively described by a two-state stochastic cellular automaton (sCA) [6] with spatial discretization provided by the mesoscopic skin scales and with time steps representing increasing time intervals as the lizard grows. Note that spatial and color-state discretizations both emerge from the superposition of the RD system with the lizard's skin

Published by the American Physical Society under the terms of the Creative Commons Attribution 4.0 International license. Further distribution of this work must maintain attribution to the author(s) and the published article's title, journal citation, and DOI. geometry [6,7]: Diffusion is reduced at the thin borders of much thicker skin scales such that it homogenizes color within a scale, while color-state transitions occur at sharp scale borders. The flipping probabilities of the sCA [Fig. 1(b)] were inferred in Ref. [6] by monitoring the skin patterns of individuals over a period of several years. Computer simulations of the sCA yield patterns very similar to those of real lizards [6] [Fig. 1(c)] within the corresponding number of time steps $(\sim 10-15)$. The lizard sCA is an example of an ergodic Markov chain on the space of possible patterns, admitting a stationary probability distribution characterizing the late-time patterns.

The celebrated Ising model $[8,9]$, whose states can be generated dynamically, can be viewed as a simple selforganized pattern production mechanism. It was discovered to describe a wide range of processes in physics (idealized but also real magnetic materials and gases $[8,10,11])$, chemistry (reaction-diffusion systems [12]), biology (for modeling population dynamics [13], neural circuits [14], glioma invasion [15-17], protein folding [18], gene regulation [19], and DNA compaction [20]), and even economic and social sciences (e.g., Ref. [21]). Here, we evaluate the efficiency of the Ising model to describe the skin color patterning of the ocellated lizard. This description inevitably focuses on how measurable interactions among mesoscopic skin scales explain the emergence of the macroscopic skin color patterns while ignoring the "lower-level" processes. Although our study does not provide insight in terms of cell biology, it suggests that natural selection can efficiently act upon self-organizational processes even when they maximize the Gibbs entropy.

The Ising model in thermal equilibrium is characterized by a stationary probability distribution given by the canonical ensemble (or Gibbs measure). Its time evolution 


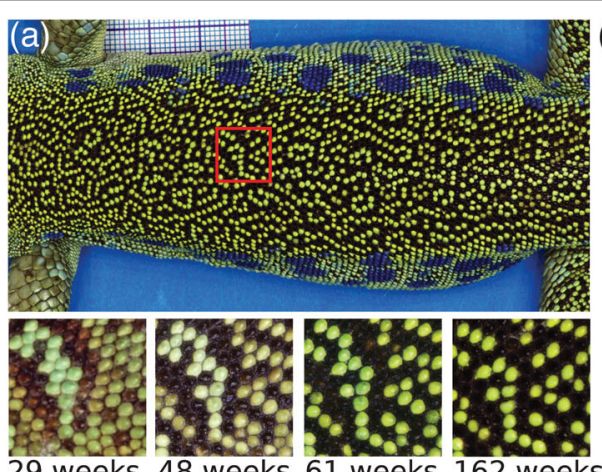

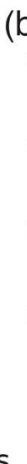

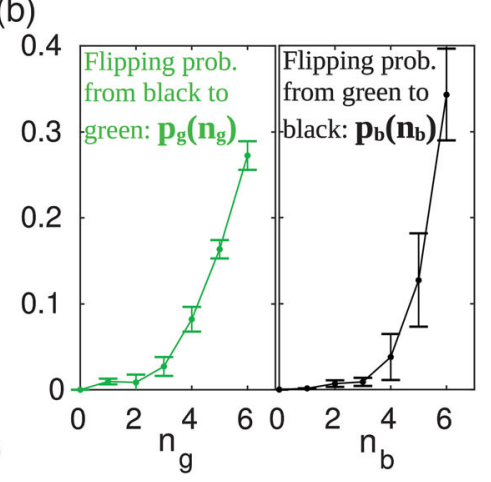

(c)

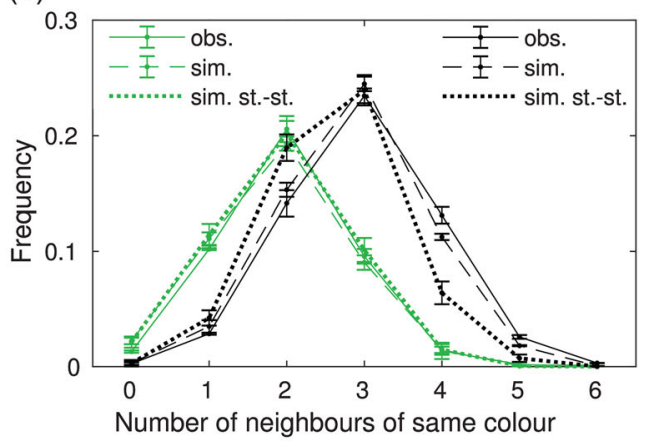

FIG. 1. (a) Dorsal pattern of an adult T. lepidus and time evolution for a detailed region. (b) Probabilities $p_{g, b}\left(n_{g, b}\right)$ of scale color flipping as a function of the number of green $\left(n_{g}\right)$ or black $\left(n_{b}\right)$ nearest neighbors (Supplemental Table 1.1 [1]). Uncertainty bars indicate standard deviation for a sample of ten time points in three individuals. (c) Relative frequencies of green scales (dashed green lines) and black scales (dashed black lines) as a function of the neighborhood: comparison of observed data from three adult lizards against CA simulations using $p_{g}\left(n_{g}\right)$ and $p_{b}\left(n_{b}\right)$ from (b) (dotted black line, steady-state regime).

is often expressed as a Monte Carlo Markov chain (MCMC), which also acts as a sampler of the canonical ensemble. Its transition probabilities are usually given by the "heat-bath algorithm," which adapts Glauber dynamics [22] to the discrete-time setup. The questions we study here are (1) Can we fix the Ising model parameters so that the canonical probability distribution is equal, or very close, to the stationary probability distribution of the lizard sCA Markov chain? (2) Is there an MCMC for the Ising model whose dynamics reproduce well that of the lizard sCA? Concerning question 1, we find that the Ising model for the lizard sCA is an antiferromagnet at finite temperature, with an external magnetic field accounting for the asymmetry of black and green scale frequencies. The Ising model description of color patterning is remarkable in two respects. First, the two-state degree of freedom (green or black) at a mesoscopic scale is enough for an effective description of the underlying continuous microscopic mechanisms. Second, the Ising model is in the antiferromagnetic phase on a triangular lattice, which is essentially a frustrated system, illustrating the idea that self-organized complexity arises from frustration [23,24]. Concerning question 2, we find that the dynamics reproducing the lizard sCA are different from Glauber dynamics. Let us note that an arbitrary set of sCA flipping probabilities is not necessarily associated to an Ising model, making our results nontrivial.

We also comment on the Ising model in the lowtemperature limit and its potential as a pattern-generation mechanism for an idealized lizard.

The stochastic cellular automaton as a Markov chain.The hexagonal scales on the back of the lizard are modeled by vertices of a finite triangular lattice of $N=$ a few thousand sites; i.e., the lattice defects (10\%-15\% of scales) are neglected. In simulations, periodic boundary conditions are imposed so that interactions with the flanks, head, and tail are ignored. Each scale $k$ is in a state $\sigma_{k}$ which can take two values: +1 for green and -1 for black. A pattern $\sigma=$ $\left(\sigma_{1}, \ldots, \sigma_{N}\right)$ is one of the $2^{N}$ possible configurations of scale states. Probability distributions on the set of patterns will be denoted by $\pi$. Its time evolution is approximated by a discrete-time Markov chain with transition probability matrix $P\left(\sigma \mid \sigma^{\prime}\right)$ giving the probability of transition from $\sigma^{\prime}$ to $\sigma$ in a single step:

$$
\pi^{(t+1)}(\sigma)=\sum_{\sigma^{\prime}} P\left(\sigma \mid \sigma^{\prime}\right) \pi^{(t)}\left(\sigma^{\prime}\right) .
$$

In the case of the lizard sCA, $P\left(\sigma \mid \sigma^{\prime}\right)$ can be computed from the flipping probabilities $p_{g, b}\left(n_{g, b}\right)$ in Fig. 1(b). In practice, it is a very large matrix of size $2^{N} \times 2^{N}\left(\approx 10^{1806}\right.$ for $N=$ 3000 scales). We assume that this Markov chain is ergodic, which could, in principle, be shown by studying the nonzero entries of $P$. In that case, the stationary probability distribution $\pi_{\text {lizard }}$ satisfying

$$
\pi_{\text {lizard }}(\sigma)=\sum_{\sigma^{\prime}} P\left(\sigma \mid \sigma^{\prime}\right) \pi_{\text {lizard }}\left(\sigma^{\prime}\right)
$$

is attractive and gives the late-time probability distribution of the patterns of T. lepidus. We further assume (and confirm below) that the mixing time of this Markov chain is not much larger than a few tens of time steps, i.e., the life span of the lizard.

The Ising model.-The probability distribution for the canonical ensemble of the Ising model is given by

$$
\pi_{\text {Ising }}(\sigma)=\frac{1}{Z} \exp \left(\beta J \sum_{\left\langle k, k^{\prime}\right\rangle} \sigma_{k} \sigma_{k^{\prime}}+\beta B \sum_{k} \sigma_{k}\right),
$$

where $J$ is the link energy, $B$ is the site energy, and $\beta$ is the reciprocal temperature. The first sum is over all pairs of nearest neighbors. The factor $Z$ is the partition function normalizing the probabilities, known only as a closed formula in some special cases, e.g., for $B=0$ [25-27]. 
Monte Carlo Markov chains for the Ising model.-To find parameters $\beta J$ and $\beta B$ for which the probability distributions $\pi_{\text {lizard }}$ and $\pi_{\text {Ising }}$ are very close, we use the Metropolis-Hastings algorithm [28,29], which is an MCMC admitting $\pi_{\text {Ising }}$ as its attractive stationary steady-state distribution. Its transition matrix is

$$
P_{\mathrm{MCMC}}\left(\sigma \mid \sigma^{\prime}\right)=g\left(\sigma \mid \sigma^{\prime}\right) A\left(\sigma, \sigma^{\prime}\right), \quad \sigma \neq \sigma^{\prime},
$$

where $g\left(\sigma \mid \sigma^{\prime}\right)$ is the probability to select $\sigma$ as the next state when the current state is $\sigma^{\prime}$ and $A\left(\sigma, \sigma^{\prime}\right)$ is the probability to accept it. The diagonal elements are defined using $\sum_{\sigma} P_{\mathrm{MCMC}}\left(\sigma \mid \sigma^{\prime}\right)=1$.

Using (i) the assumption of detailed balance, (ii) a "single-flip" scheme for the state selection process $g\left(\sigma \mid \sigma^{\prime}\right)$, and (iii) a special ansatz for the dependence of the state acceptance probability $A\left(\sigma, \sigma^{\prime}\right)$, it can be shown (Supplemental Material [1]) that the following flipping probabilities

$$
\begin{aligned}
& p_{g}^{\operatorname{MCMC}}\left(n_{g}\right)=\mathcal{A}\left(e^{-2 \beta J\left(2 n_{g}-6\right)-2 \beta B}\right), \\
& p_{b}^{\operatorname{MCMC}}\left(n_{b}\right)=\mathcal{A}\left(e^{-2 \beta J\left(2 n_{b}-6\right)+2 \beta B}\right)
\end{aligned}
$$

for a certain function $\mathcal{A}(R)$ define a single-flip MCMC whose stationary probabilistic distribution is precisely $\pi_{\text {Ising. }}$. A sufficient condition on $\mathcal{A}(R)$ is that it satisfies $\mathcal{A}\left(\mathbb{R}^{>0}\right) \subset(0,1]$ and the functional relation

$$
\mathcal{A}(1 / R)=\mathcal{A}(R) / R .
$$

The lizard Ising model.-First, we determine the best Ising parameters describing the lizard sCA independently from $\mathcal{A}$. Equations (5) and (6) imply the following relation:

$$
\log \frac{p_{g}^{\mathrm{MCMC}}(n)}{p_{b}^{\mathrm{MCMC}}(6-n)}=(-4 \beta J) n+(12 \beta J-2 \beta B) .
$$

This is an affine function of $n$. The corresponding quantity $\log p_{g}(n) / p_{b}(6-n)$ for the lizard flipping probabilities is plotted in Fig. 2(a), together with the best least-squares fit for $a n+b$. From the fitted coefficients $a$ and $b$, values for the Ising model parameters $\beta J=-a / 4$ and $\beta B=$ $-(3 a+b) / 2$ can be extracted:

$$
\beta J=-0.457 \pm 0.016, \quad \beta B=-0.51 \pm 0.14 .
$$

This is an antiferromagnetic Ising model $(J<0)$ at finite temperature with asymmetry favoring black scales $(B<0)$. The affine fit being close to the lizard data, the Ising model probability distribution $\pi_{\text {Ising }}$ with these parameters should approximate well the lizard sCA stationary probability distribution $\pi_{\text {lizard }}$. A direct comparison for small lattices (Supplemental Fig. 3.1 [1]) and numerical simulations for larger lattices [Figs. 2(b) and 2(c)] show that the Ising (a)

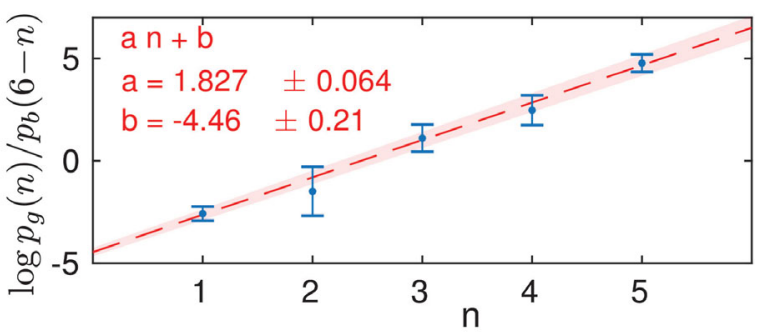

(b)

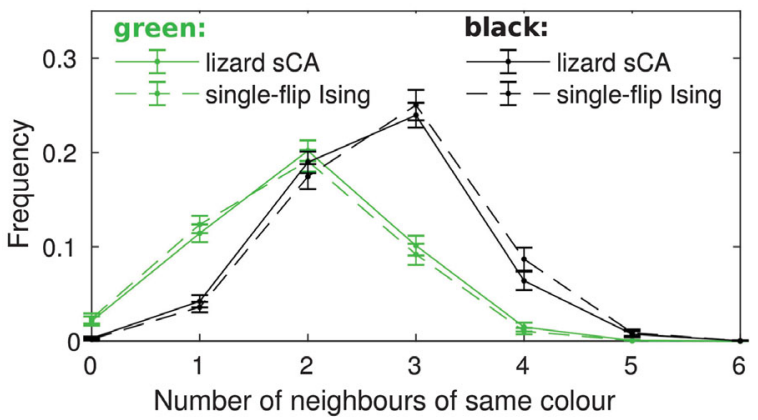

(c)

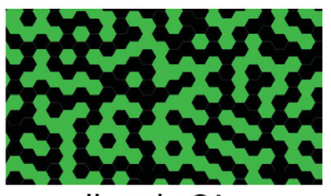

lizard SCA

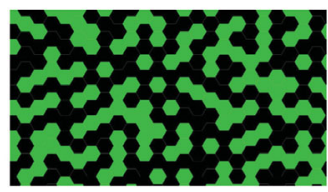

single-flip Ising
FIG. 2. (a) Values of $\log p_{g}(n) / p_{b}(6-n)$ from the data in Fig. 1(b) with best affine fit (red dotted line and shading for uncertainty). Values at $n=0$ and 6 cannot be reliably inferred from the available data. (b) Nearest-neighbor statistics: relative frequencies of green and black scales as a function of the neighborhood [data from 100 runs of 5000 time steps for both sCA and single-flip MCMC fitted Ising model with $\mathcal{A}(R)=$ $R /(R+1)$; initial condition in Supplemental Fig. 2.2 [1]]. (c) Typical patterns obtained in (b).

model generates nearest-neighbor statistics and typical patterns similar to those obtained with the sCA at late times.

Second, we determine the function $\mathcal{A}$ for which the flipping probabilities (5) are closest to those of the lizard sCA reproduced in Fig. 1(b). The only 14 values of $R$ where $\mathcal{A}(R)$ matters are

$$
R=R_{n}:=\exp [-2 \beta J(2 n-6)-2 \beta B]
$$

and $R=1 / R_{n}$ for $n=0, \ldots, 6$. Since from Eq. (6) we have $\mathcal{A}\left(1 / R_{n}\right)=\mathcal{A}\left(R_{n}\right) / R_{n}$, we have seven degrees of freedom to fix, namely, $\mathcal{A}_{n}:=\mathcal{A}\left(R_{n}\right)$. These can be fixed by minimizing the sum of squared differences

$$
\sum_{c=g, b} \sum_{n=0}^{6}\left[p_{c}(n)-p_{c}^{\mathrm{MCMC}}(n)\right]^{2}
$$

Using Eq. (5), we find 
(a)

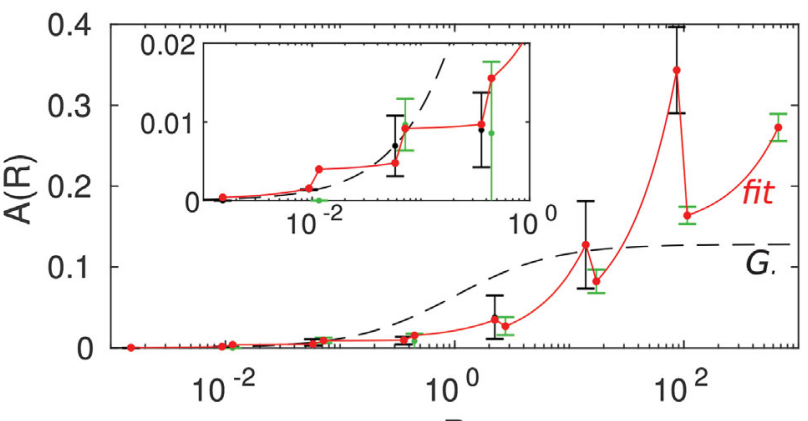

(b)

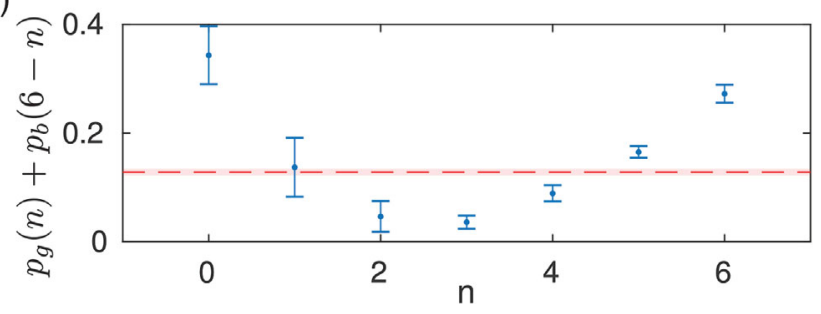

FIG. 3. (a) Function $\mathcal{A}(R)$ obtained from the fit (red points and linear interpolation in red lines) and comparison with the lizard sCA flipping probabilities reported from Fig. 1(b). Dashed line, Glauber function $\mathcal{A}_{G}(R)$ with $c$ fixed below. (b) Values of $p_{g}(n)+p_{b}(6-n)$ from the data in Fig. 1(b) with best constant fit $c$ (red).

$$
\mathcal{A}_{n}=\left[R_{n}^{2} p_{g}(n)+R_{n} p_{b}(6-n)\right] /\left(R_{n}^{2}+1\right),
$$

which are shown in Fig. 3(a). These lead to the following Ising model flipping probabilities:

$$
p_{g}^{\mathrm{MCMC}}\left(n_{g}\right)=\mathcal{A}_{n_{g}}, \quad p_{b}^{\mathrm{MCMC}}\left(n_{b}\right)=\frac{\mathcal{A}_{6-n_{b}}}{R_{6-n_{b}}} .
$$

The numerical values represented in Fig. 3(a) (and Supplemental Table 1.2 [1]) are very close to the lizard sCA flipping probabilities.

The dynamics of the two models also compare well. For example, the average number of flips as a function of time are similar (Supplemental Fig. 5.1 [1]) when a single CA generation is identified to $N$ single flips. We conclude that the function $\mathcal{A}$ defined by the values $\mathcal{A}_{n}$ and $\mathcal{A}_{n} / R_{n}$ gives a very good approximation of the skin color change dynamics in T. lepidus. Dynamical simulations also seem to indicate a posteriori that both the lizard sCA and the Ising MCMC with fitted flipping probabilities are indeed ergodic with mixing time of the order of a few tens of CA time steps.

Next, we compare the dynamics obtained above to Glauber dynamics traditionally used to describe relaxation of the Ising model toward thermal equilibrium [22]. For discrete-time evolution of single flips, the Glauber dynamics can be defined by $\mathcal{A}(R)=\mathcal{A}_{G}(R):=c R /(R+1)$ for flipping rate $c$. The function $\mathcal{A}_{G}(R)$ obeys an extra functional relation: $\mathcal{A}_{G}(R)+\mathcal{A}_{G}(1 / R)=c$. Therefore, fixing $n=n_{g}=6-n_{b}$, the flipping probabilities built using $\mathcal{A}_{G}$ should satisfy $p_{g}(n)+p_{b}(6-n)=c$ for $n=0,1, \ldots, 6$, independently of $\beta J$ and $\beta B$. As shown in Fig. 3(b), this relation is not satisfied by the lizard sCA flipping probabilities, showing that Glauber dynamics is unsuited for modeling the scale color dynamics.

Skin patterns and the Ising model in the low-temperature limit.-The lizard skin scale color flipping process is stochastic. This randomness could be a consequence of different factors affecting an otherwise deterministic process: geometrical inhomogeneities and defects of the lattice of sites, longer-range interactions, simultaneous flipping of nearest neighbors, the two-state approximation, some unknown microscopic degrees of freedom, etc. Let us here assume that all these nuisance effects can be neglected in an idealized lizard. If a corresponding Ising model exists, it should be in the limit of zero temperature $(\beta \rightarrow \infty)$, since temperature is related to random fluctuations in the Ising model.

In this regime, $\pi_{\text {Ising }}$ in Eq. (3) is vanishing everywhere except for ground-state patterns minimizing the energy

$$
E=-J \sum_{\operatorname{links}\left\langle k, k^{\prime}\right\rangle} \sigma_{k} \sigma_{k^{\prime}}-B \sum_{\text {sites } k} \sigma_{k},
$$

over which it is uniform. The phase space of ground-state patterns of the Ising model on the triangular lattice is well known $[26,30]$. It is represented in Fig. 4, with the lowtemperature limit $(\beta \rightarrow \infty)$ lying on the outer circle. In that limit, there are eight regions giving qualitatively different ground-state patterns (see Supplemental Material [1], which includes Ref. [31]).

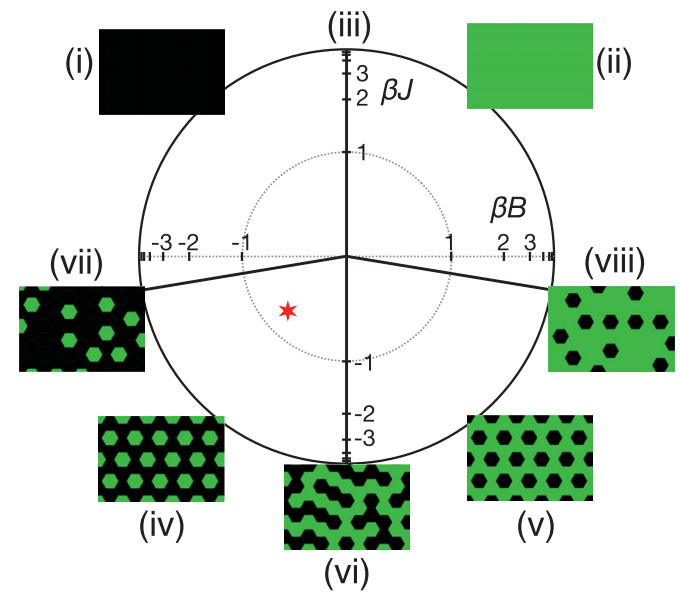

FIG. 4. Parameter space of the triangular Ising model in polar coordinates with radial coordinate mapped to a finite range. The zero temperature limit is the outer circle, where we indicate the eight regions with different types of ground-state patterns. The system exhibits order in regions (i)-(v) and disorder at points (vi)-(viii) (see Supplemental Material [1]). The red star indicates the location of the optimized lizard Ising model at finite temperature. 
The first five [(i)-(v)] are said to be "ordered" phases in the low-temperature limit, since the number of ground-state patterns does not scale with the lattice size $N$. In a dynamical setup, these ground-state patterns may not be relevant for patterning, because the relaxation time of the system may be exponential in the system size. Some metastable states may even be stationary for single-flip dynamics, as ergodicity often does not hold for the corresponding MCMC.

The last three [(vi)-(viii)] are said to be "disordered" phases in the low-temperature limit, since the number of ground-state patterns scales exponentially with the lattice size $N$ [26,32]. These may be the most relevant phases for patterning: Variability of the pattern is still maintained even as the source for stochasticity represented by temperature is lowered. Among these three, the frustrated antiferromagnetic point (vi) $(B=0, J<0)[26]$ arguably gives the most relevant patterns for T. lepidus (Supplemental Fig. 6.1 [1]).

Conclusions. - Starting from the description of Timon lepidus pattern dynamics as a sCA, we evaluated the probability of finding a given pattern in terms of the Ising model canonical probability distribution. The corresponding Ising model is in the antiferromagnetic phase at finite temperature. The pattern evolution can be well approximated by a single-flip Monte Carlo Markov chain describing the thermalization of the Ising model. We found, however, that the optimal dynamics are not those of Glauber. We also discussed the patterns generated in the zero temperature limit, especially in terms of pattern variability.

This new angle on the study of animal skin patterning does not provide much insight on the underlying microscopic cell-biology processes [6,7]. However, it poses unconventional questions on some possible relationships between genetic variability, the Darwinian selection process, and the entropy maximization principle. Indeed, for a given local interaction model, the canonical probability distribution over patterns maximizes the Gibbs entropy $-\sum_{\sigma} \pi(\sigma) \log \pi(\sigma)$ under the constraint that the mean "energy" of the pattern is fixed. In this way, the energy of a pattern becomes a well-defined notion for the relevant local interaction model. We have shown here that this concept works for the skin color patterning process in $T$. lepidus if this energy is proportional to the standard Ising model energy in the antiferromagnetic phase.

In light of these results, it may be argued that, at a given Ising energy, the mesoscopic dynamical system can generate a large number of configurations of sites (i.e., a large number of macroscopic patterns) that are equivalent in terms of their corresponding fitnesses. In other words, the reproductive success that a scale-by-scale color pattern confers to an animal is likely associated to the general quality of the pattern-e.g., as defined by the nearestneighbor statistics [Figs. 1(c) and 2(b)] and corresponding to the Ising energy - rather than by its exact specific configuration. Hence, the Ising model framework illuminates the fact that Gibbs entropy maximization does not preclude the patterning process to remain highly "evolvable": Stochastic genetic variations (generated by mutations) have the potential to affect the Ising link and site energies, such that multiple kinds of patterns (Fig. 4) can be explored, while natural selection drives the system to a certain mean energy related to the qualitative features of the patterns (see Supplemental Fig. 7.1 [1]). Concomitantly, the patterning process would nevertheless remain robust at all times of this evolutionary exploration of the $(\beta J, \beta B)$ phase space, because stochasticity generated by entropy maximization at a given energy of the system is irrelevant to selection.

We suggest that this combination of "evolvability" and "robustness" is nontrivial given the strong effective stochastic (entropic) component of the patterning processes we study here.

The perspective of making local interaction models more general descriptions of color patterning processes could be tested by studying other species exhibiting skin scale flipping dynamics but whose steady-state patterns (i) are qualitatively different from those observed in T. lepidus and (ii) would still follow a canonical probability distribution. Another avenue of further study is to clarify how cellbiology parameters affect scale-color flipping probabilities and in which of these cases fitting of the Ising model remains possible. Such a study would link three spatial scales: the "microscopic" interactions among cells, the flipping probabilities of mesoscopic skin scales, and the "macroscopic" description of skin color patterns.

This work was supported by grants to M. C. M. from the G. \& A. Claraz foundation, the Swiss National Science Foundation (FNSNF, Grants No. 31003A_179431 and No. CR32I3_162743), the International Human Frontier Science Program Organisation (HFSP RGP0019/2017), and the European Research Council (ERC, Advanced Grant EVOMORPHYS) under the European Union's Horizon 2020 research and innovation program. This work was also supported by grants to S.S. from the Swiss National Science Foundation (FNSNF, Grant No. 197226) and National Center of Competence in Research (NCCR SwissMAP).

* Corresponding author. michel.milinkovitch@unige.ch

[1] See Supplemental Material at http://link.aps.org/ supplemental/10.1103/PhysRevLett.128.048102 for supplemental details on methods and results.

[2] I. C. Cuthill, M. Stevens, J. Sheppard, T. Maddocks, C. A. Párraga, and T.S. Troscianko, Disruptive coloration and background pattern matching, Nature (London) 434, 72 (2005). 
[3] M. Stevens, I. S. Winney, A. Cantor, and J. Graham, Outline and surface disruption in animal camouflage, Proc. R. Soc. B 276, 781 (2009).

[4] R. Bu, F. Xiao, P. G. Lovell, Z. Ye, and H. Shi, Structural and colored disruption as camouflage strategies in two sympatric Asian box turtle species (Cuora spp.), Global Ecol. Conserv. 24, e01361 (2020).

[5] A. M. Turing, The chemical basis of morphogenesis, Phil. Trans. R. Soc. B 237, 37 (1952).

[6] L. Manukyan, S. A. Montandon, A. Fofonjka, S. Smirnov, and M.C. Milinkovitch, A living mesoscopic cellular automaton made of skin scales, Nature (London) 544, 173 (2017).

[7] A. Fofonjka and M. C. Milinkovitch, Reaction-diffusion in a growing 3D domain of skin scales generates a discrete cellular automaton, Nat. Commun. 12, 2433 (2021).

[8] W. Lenz, Beiträge zum Verständnis der magnetischen Eigenschaften in festen Körpern, Phys. Z. 21, 613 (1920).

[9] E. Ising, Beitrag zur Theorie des Ferromagnetismus, Z. Phys. 31, 253 (1925).

[10] C. N. Yang and T. D. Lee, Statistical theory of equations of state and phase transitions. I. Theory of condensation, Phys. Rev. 87, 404 (1952).

[11] J. C. Wright, H. W. Moos, J. H. Colwell, B. W. Mangum, and D. D. Thornton, $\mathrm{DyPO}_{4}$ : A Three-Dimensional Ising Antiferromagnet, Phys. Rev. B 3, 843 (1971).

[12] M. Merle, L. Messio, and J. Mozziconacci, Turing-like patterns in an asymmetric dynamic Ising model, Phys. Rev. E 100, 042111 (2019).

[13] A. E. Noble, J. Machta, and A. Hastings, Emergent longrange synchronization of oscillating ecological populations without external forcing described by Ising universality, Nat. Commun. 6, 6664 (2015).

[14] E. Schneidman, M. J. Berry, R. Segev, and W. Bialek, Weak pairwise correlations imply strongly correlated network states in a neural population, Nature (London) 440, 1007 (2006).

[15] B. M. Rubenstein and L. J. Kaufman, The role of extracellular matrix in glioma invasion: A cellular potts model approach, Biophys. J. 95, 5661 (2008).

[16] E. Khain, C. M. Schneider-Mizell, M. O. Nowicki, E. A. Chiocca, S. E. Lawler, and L. M. Sander, Pattern formation of glioma cells: Effects of adhesion, Europhys. Lett. 88, 28006 (2009).
[17] S. Torquato, Toward an Ising model of cancer and beyond, Phys. Biol. 8, 015017 (2011).

[18] T. Aksel and D. Barrick, Analysis of repeat-protein folding using nearest-neighbor statistical mechanical models, Methods Enzymol. 455, 95 (2009).

[19] N. Santhanam, J. Dingel, and O. Milenkovic, in Proceedings of the 2009 IEEE Information Theory Workshop on Networking and Information Theory (IEEE, New York, 2009), pp. 156-160, 10.1109/ITWNIT.2009.5158562.

[20] N. N. Vtyurina, D. Dulin, M. W. Docter, A. S. Meyer, N. H. Dekker, and E. A. Abbondanzieri, Hysteresis in DNA compaction by Dps is described by an Ising model, Proc. Natl. Acad. Sci. U.S.A. 113, 4982 (2016).

[21] G. Zaklan, F. Westerhoff, and D. Stauffer, Analysing tax evasion dynamics via the Ising model, J. Econ. Interact. Coord. 4, 1 (2009).

[22] R. J. Glauber, Time-dependent statistics of the Ising model, J. Math. Phys. (N.Y.) 4, 294 (1963).

[23] Y. I. Wolf, M. I. Katsnelson, and E. V. Koonin, Physical foundations of biological complexity, Proc. Natl. Acad. Sci. U.S.A. 115, E8678 (2018).

[24] M. I. Katsnelson, Y. I. Wolf, and E. V. Koonin, Towards physical principles of biological evolution, Phys. Scr. 93, 043001 (2018).

[25] L. Onsager, Crystal statistics. I. A two-dimensional model with an order-disorder transition, Phys. Rev. 65, 117 (1944).

[26] G. H. Wannier, Antiferromagnetism. The triangular Ising net, Phys. Rev. 79, 357 (1950).

[27] R. Houtappel, Order-disorder in hexagonal lattices, Physica (Amsterdam) 16, 425 (1950).

[28] N. Metropolis, A. W. Rosenbluth, M. N. Rosenbluth, A. H. Teller, and E. Teller, Equation of state calculations by fast computing machines, J. Chem. Phys. 21, 1087 (1953).

[29] W. K. Hastings, Monte Carlo sampling methods using Markov chains and their applications, Biometrika 57, 97 (1970).

[30] B. Metcalf, Ground state spin orderings of the triangular Ising model with the nearest and next nearest neighbor interaction, Phys. Lett. 46A, 325 (1974).

[31] R. J. Baxter, Hard hexagons: exact solution, J. Phys. A 13, L61 (1980).

[32] R. J. Baxter and S. K. Tsang, Entropy of hard hexagons, J. Phys. A 13, 1023 (1980). 